
allemande

\title{
S'opposer à la destruction de la nature alpestre : Meinrad Inglin ou la résistance littéraire
}

\section{Régine Battiston}

\section{(2) OpenEdition}

1 Journals

Édition électronique

URL : https://journals.openedition.org/allemagne/2253

DOI : 10.4000/allemagne.2253

ISSN : 2605-7913

Éditeur

Société d'études allemandes

\section{Édition imprimée}

Date de publication : 1 juillet 2020

Pagination : 99-114

ISSN : 0035-0974

\section{Référence électronique}

Régine Battiston, « S'opposer à la destruction de la nature alpestre : Meinrad Inglin ou la résistance littéraire », Revue d'Allemagne et des pays de langue allemande [En ligne], 52-1 | 2020, mis en ligne le 06 juillet 2021, consulté le 19 février 2022. URL : http://journals.openedition.org/allemagne/2253 ; DOI : https://doi.org/10.4000/allemagne.2253 


\title{
Lectures littéraires
}

\section{S’opposer à la destruction de la nature alpestre: Meinrad Inglin ou la résistance littéraire}

\author{
- Régine Battiston*
}

Meinrad Inglin (Schwyz, 1893-1971) campe tous ses récits dans les Alpes suisses. Auteur du terroir suisse, habité par une croyance animiste et panthéiste (sans pour autant négliger la transcendance), il est tout à la fois dissident et écologiste avant l'heure, très soucieux de la préservation des sites, de la faune et de la flore de son pays natal (il a vécu toute sa vie dans sa ville natale de Schwyz). Il oppose la vie dans la nature à la civilisation technicienne, stigmatise les excès de celle-ci, en montrant que l'arrogance de l'homme met son cadre de vie en péril. Citoyen militant engagé dans des actions de manifestations contre la construction de barrages et de routes en Suisse dans les années 1945-60, il a pris part dans son engagement pour la préservation de l'environnement à des pétitions contre la construction de centrales hydroélectriques en Engadine et sur le Rhin, de téléphériques qui gâchent le paysage, de routes qui facilitent l'accès aux touristes à des sites naturels préservés et inaccessibles. Il oriente très tôt son œuvre vers la mise en scène de catastrophes induites par l'action de l'homme sur les paysages (par exemple l'incendie dans Grand Hôtel Excelsior, 1928). Dans le roman Urwang (1954), il montre les conséquences catastrophiques de l'inondation d'une vallée pour la construction d'un barrage et d'une centrale hydroélectrique; il y détaille les phases de l'expropriation des paysans à qui la nouvelle situation sera très défavorable et dénonce la disparition pure et simple de la faune et de la flore sans contrepartie. Urwang marque un tournant dans son œuvre, Inglin démontrant que l'individu est quantité négligeable quand des intérêts financiers sont en jeu. Le ton pragmatique et désabusé et le style sobre soulignent que tout combat de ce type est perdu d'avance, mais le roman montre également que l'homme n'est pas une marchandise.

Dans les années d'après-guerre (1945 à 1960), l'Europe ainsi que la Suisse connaissent un développement industriel et économique considérable qui implique, tout comme l'augmentation de la population et du confort de vie (vers une démocratisation de

* Professeur à l'Université de Haute-Alsace - Mulhouse, ILLE UR 4363. 
l'automobile entre autres), une augmentation de la consommation énergétique. La Suisse se lance dans une série de grands travaux de construction de barrages notamment dans l'Engadine, en noyant des vallées alpestres et en déplaçant la population faite essentiellement de paysans pauvres. Une campagne de presse régulière (dont le journal Neue Zürcher Zeitung, NZZ) explique de façon détaillée et documentée avec force aspects techniques (en donnant la parole à des experts) la nécessité de produire plus d'énergie, afin que le pays reste au maximum indépendant en limitant son importation. La Suisse choisit de développer d'abord l'énergie hydroélectrique et se tournera vers le nucléaire dans les années 1970. De 1950 aux années 1970, on a construit 27 barrages hydroélectriques dans le canton des Grisons, et 63 dans le reste de la Suisse (le chiffre paraît exorbitant par rapport à la taille du pays). La conjoncture était favorable à ces constructions, mais elle provoqua aussi une prise de conscience salutaire en matière de protection de l'environnement. En 1943, deux consortiums en Bas-Engadine déposèrent un projet de construction de plusieurs centrales hydroélectriques. Les opposants se constituèrent en association de défense des sites naturels, dont la fameuse association «Lia Naira ${ }^{(1)}$.

Durant cette période, le tourisme commence à beaucoup se développer et l'arrivée en masse de touristes va mettre en péril des sites d'écosystèmes fragiles, notamment avec les marcheurs en montagne qui cueillent aussi des fleurs rares, telles l'edelweiss, la gentiane, etc. L'œuvre de maturité de Meinrad Inglin s'inscrit précisément dans cette période.

L'écrivain a toujours vécu très proche de la nature, dans ses œuvres l'homme est un être minuscule comparé à l'immensité des paysages alpins (cf. Die Graue March, $1935)^{(2)}$. Dans tous ses scénarios, c'est toujours elle qui l'emporte, notamment de par son relief, ses accès difficiles, les aléas d'un climat rude. La difficile survie humaine par l'isolement dans la montagne est soumise à un quotidien fait de labeur et de dépendance à l'environnement et aux animaux domestiques. Dès les débuts littéraires d'Inglin, la thématique de la nature s'impose d'elle-même comme un fil rouge de son œuvre. Ainsi, après avoir montré la beauté de la nature sauvage et les aléas de la vie humaine en haute montagne (avec ses accidents nombreux ${ }^{(3)}$ ), la défense de la nature

1 Dans ce contexte est apparue l'association Lia naira (en ladin «qui se rencontrent la nuit», car ce fut le mode de fonctionnement des militants) qui engagea des initiatives citoyennes contre la construction de ces barrages, notamment sur les fleuves Inn et Spöl (en direction de l'Autriche). Elle se rapprocha du «Comité Rheinau » qui avait sur le Rhin des visées analogues - voir plus bas. Dans leurs actions, la majorité des signatures venait des cantons de Zurich et de Berne, le noyau des activistes était constitué de personnes issues du monde académique (voir NZZ du 02.08.1957). Leur but était de bloquer la construction des nombreux barrages en projet. Cependant leur tâche fut difficile, car les promoteurs du projet avaient prévu en compensation d'augmenter de deux tiers la surface du parc national de l'Engadine, mais en sacrifiant le fleuve Spöl en échange (ce qui ressemble à une forme de chantage et de corruption). Selon la manière dont on fait les calculs de surfaces gagnées pour le parc, le gain n'est que de $10 \%$, car des surfaces agricoles furent transformées en surfaces sauvages (et donc perdues pour l'agriculture).

2 On pourra se référer au site Internet de la Fondation Meinrad Inglin: www.meinradinglin.ch (25.06.2019).

3 Le père d'Inglin est décédé en 1906 dans un accident de ce type (chute en montagne). Son décès prématuré a pesé sur le devenir du fils et le décès de sa mère, alors qu’il n’a que 17 ans, précipitera le jeune Inglin dans une période de doutes et d'errance, qui aura pour conséquence une formation professionnelle chaotique. 
devient logiquement le cheval de bataille de l'œuvre littéraire d'un homme du cru, attaché à son paysage natal, malgré des tentatives de vie citadine avortées (comme lors de ses études à Berne, Genève et Neuchâtel ou sa tentative de prendre pied à Berlin comme journaliste en 1922 et pour trouver un éditeur). La défense de la nature en politique devient son horizon d'attente.

Inglin reste l'homme de sa petite ville, amateur de longues marches dans la montagne et de parties de chasse (dont il s'éloignera peu à peu, le chien fidèle restant alors le prétexte de sorties pédestres ${ }^{(4)}$. Lors de ses randonnées botaniques avec son ami Hans von Matt, un de leurs sujets de prédilection reste la littérature et leur relation à leur patrie:

«Wir wanderten auf dem feuchten kühlen Waldweg weiter, bis wir einen besonnten Platz fanden, wo liegende Baumstämme zum Sitzen einluden. Wir schauten einige Zeit den treibenden Wolken zu. Dann glitt das Gespräch - wie gewohnt - vom botanischen zum literarischen Stoff hinüber. Ein Thema, das uns beide immer beschäftigte, war unsere Beziehung zur Heimat. Der Zug ins Internationale, der in Kunst und Literatur immer spürbar wurde, zwang uns, das Thema neu zu überdenken. Meinrad empfand die Gefahr, ins Volkstümliche abzusinken, als ebenso groß wie das drohende Verflachen im Internationalen. Man müsse allerdings - meinte er - fremde Länder und Menschen kennengelernt haben, um die Heimat voll zu schätzen ${ }^{(5)}$.

Par patrie, on peut comprendre chez Inglin l'attachement à sa région et à ses paysages magnifiques et gigantesques. Dans les notes qu'il a laissées dans son fonds d'archives à Schwyz ${ }^{(6)}$, on remarque d'emblée son intérêt très précoce (qui a gagné en importance avec l'âge) pour le développement géologique de la terre, de sa région alpine, de la Suisse tout entière. Des années 1930 à la fin de sa vie, il a amassé des coupures de presse sur ce thème. Les sujets qui y apparaissent sont entre autres la conservation des paysages (roches, botanique, animaux) et l'évolution géologique du paysage ${ }^{(7)}$.

Avec le temps, il s'engagera dans différentes associations, dont celle de la protection des animaux du canton de Schwyz ${ }^{(8)}$, et également dans la commission de protection de la nature et de la patrie du canton de Schwyz, dont il est secrétaire (Natur- und Heimatschutzkommission des Kantons Schwyz $\left.{ }^{(9)}\right)$. Il est aussi membre de la «Sektion Mythen"

4 Voir sur l'abandon de la chasse le témoignage de Hans von Matт, Wanderungen mit Meinrad Inglin, Einsiedeln/Zurich/Cologne, Benziger, 1978, p. 39. À la fin de sa vie, Inglin ne cueillait plus de fleurs, afin de ne pas entraver le développement des plantes.

5 Ami de Hans von Matt (oncle de Peter von Matt) depuis 1927, Inglin a fait avec ce féru de botanique de nombreuses sorties en montagne, en quête de fleurs rares (telles des orchidées sauvages dont la fameuse epigogon sans feuilles - epigogium aphyllum, ou Widerbart en allemand -, seule représentante des orchidées sauvages en Europe dans le massif Arnitobel près d'Engelberg), soit pour vérifier leur existence, soit pour aider à leur réimplantation ou à leur sauvetage. Les randonnées des deux amis avec leurs épouses ont duré des années 1950 jusqu'à la mort d'Inglin en 1971, cf. photo, ibid., p. 6 et aussi, sur la botanique des orchidées, p. 9-10.

6 Nous tenons ici à remercier le Dr. Daniel Annen et la Fondation Meinrad Inglin, à la Bibliothèque cantonale de Schwyz, dans la maison Ital von Reding, pour leur invitation et la mise à disposition des archives nécessaires aux recherches pour cette étude. Nous leur exprimons ici toute notre gratitude.

7 Cf. Archives Meinrad Inglin à Schwyz, NIM 18 / Notizen 2.) 2.1.). Dans un cahier de cette même boîte, on trouve des notes manuscrites de sa main sur la carte géologique de la Suisse dès 1904 .

8 Cf. «Einladung zur Generalversammlung des 14.12.1946». Inglin est également membre du Schweizer Bund für Naturschutz, cf. Flugblatt, 7 (1956), in: NI M 18 / Notizen 2.) 2.1.)-2.4.).

9 NI M 17 Naturschutz 3.)3.8.)-3.9.). 
(située à Schwyz), affiliée au Club alpin suisse (Schweizer Alpenklub), dont l'article 1 des statuts stipule: "Sie [i.e. Die Sektion Mythen] bezweckt Gebirgswanderungen zu erleichtern, die Kenntnisse der Schweizer Alpen zu erweitern, der Erhaltung ihrer Schönheit zu dienen und die Liebe zur Heimat zu wecken und zu pflegen» $(1950)^{(10)}$. Ce programme s'applique entièrement à l'œuvre d'Inglin et à son engagement sociétal.

Le développement du tourisme alpin va fatalement appeler des modifications et un développement des infrastructures routières dans certaines vallées. Ainsi l'État fédéral (Bund) décide que la petite route Pragelstrasse dans la vallée de la Muota doit être élargie (1938). Inglin s'intéresse tant à ce sujet qu'il participera à l'action citoyenne qui s'y oppose (Initiative Pragelstrasse, voir plus bas ${ }^{(11)}$ ). Il est membre de l'association Schweizer Bund für Naturschutz, dont le bulletin couvre des thématiques diverses. Dans un de ces fascicules, on trouve la carte des vallées englouties dans le Haut-Engadine (brochure "Vom Nationalpark im Unterengadin» - Sonderdruck aus dem Jahrbuch 1957 des Vereins zum Schutz der Alpenpflanzen und Tiere. München e.V. $\left.{ }^{(12)}\right)$. Il partage ses préoccupations de défense de la nature avec ses amis (cf. sa correspondance avec le Dr. Hermann Stieger de Brunnen). Peu à peu, son intérêt et son engagement vont se concrétiser dans son œuvre.

\section{Urwang ou la vallée perdue}

Durant l'hiver 1952, Inglin collecte des signatures à Schwyz pour une pétition contre la construction de la centrale hydroélectrique de Rheinau près de Schaffhausen, en parallèle il commence à travailler au roman Urwang qui a pour sujet la destruction d'une vallée par le projet d'un barrage hydroélectrique. Pour ce faire, il se documente sur le terrain en se rendant dans la vallée de Göschenen où un tel chantier est en cours $^{(13)}$. Il parle de son projet de roman à son ami Stiefel ${ }^{(14)}$ en lui demandant de

10 Cf. NI Z 4018 - Natur 1.) Natur (allg., Naturbegriff).

11 Cf. NI M 17 - Naturschutz 1.) Inglin conserve des textes de décisions politiques y afférant («Bericht und Antrag des Bezirksrates Schwyz in die Stimmenberechtigten des Bezirks Schwyz - betreffend Bewilligung eines Bezirksbeitrages an den Bau und Unterhalt einer Pragelstrasse»).

12 Cf. NI M 17 - Naturschutz 2.)2.1.)-2.4.).

13 En 1955 commença la construction du barrage Göscheneralpsee dans le lieu du même nom au sein du canton d'Uri. Au cours des années précédentes, une mobilisation citoyenne tenta en vain de faire échouer ce projet. La construction se termina en 1959 et en 1961-62 le barrage fut mis en fonction. Mensurations de l'ouvrage: mur de retenue de $155 \mathrm{~m}$ de haut, $540 \mathrm{~m}$ de long et $700 \mathrm{~m}$ de largeur dans la base de fond. 9,3 Mio $\mathrm{m}^{3}$ de matériau de construction, volume du lac de retenue: $76 \mathrm{Mio}^{3}$, il est situé à $1792 \mathrm{~m}$ d'altitude. La route qui y mène fait $11 \mathrm{~km}$ de long, traverse la vallée du même nom et a été construite en 1944. Des habitants ont été déplacés et des maisons, ainsi que l'église, ont été inondées. Documentation complète sur: blogs.ethz.ch/crowdsourcing/2017/10/27/staudamm-goescheneralp-was-die-baudokumentation-nicht-erzaehlt/ (25.06.2019). Voir aussi la liste des lieux de Suisse qui ont été inondés pour des constructions de barrages: de.wikipedia.org/wiki/ Liste_von_durch_Stauseen_\%C3\%BCberfluteten_Orten_in_der_Schweiz (25.06.2019). Présentation actuelle par l'exploitant de l'ouvrage: www.kw-goeschenen.ch/Staudamm.73.0.html et aussi: www.kw-goeschenen.ch/Geschichte.18.0.html (25.06.2019). Cf. également la très bonne biographie d'Inglin par Beatrice von MATt, Meinrad Inglin. Eine Biographie, Zurich/Fribourg-en-Brisgau, Atlantis, 1976, p. 238.

14 «Meine nächste Erzählung wird zeigen, wie die Technik in ein hoch unberührbares Bergtal einbricht und es schließlich in einem Stausee ertränkt. (Bitte nicht weitersagen!) Du weißt, wie spielend leicht 
collecter pour lui du matériel concernant les expropriations et les réaffectations de terres aux paysans spoliés ${ }^{(15)}$. Il se pose la question du bien-fondé de cette opération d'envergure:

«Am meisten plagt mich zur Zeit die Frage, ob die steigende Energieerzeugung für uns wirklich lebensnotwendig und nicht nur eine wirtschaftliche Spekulation ist. Ich habe das Gefühl, daß die fortschreitende Industrialisierung am nationalen Kapital zehrt und unsere Substanz antastet» $(09.01 .1953)^{(16)}$.

Il tient des statistiques des vallées alpines englouties ${ }^{(17)}$, il étudie les procédés d'expropriation et les travaux menés par les ingénieurs. Il établit des cartes pour se représenter l'envergure des chantiers et les pertes de paysage naturel, il en établit de fictives pour visualiser le chantier dans son roman. Ce travail préparatoire le conforte dans son action et ses convictions. Il doute du bien-fondé du développement économique à tous crins et des sacrifices engendrés ${ }^{(18)}$. Le profit apparaît dans le roman comme une force anonyme qui broie tout sur son passage, si bien qu'il n'y a pas de gagnants, l'humanité et la Heimat lui étant sacrifiées. Il est parfois assailli de doutes sur sa capacité à mener à bien ce roman d'envergure sur un sujet si contemporain, mais son ami Stiefel le rassure sur son empathie envers la nature, sur sa capacité à comprendre les enjeux et à se poser les questions adéquates. C'est un auteur vieillissant qui entame ce chantier littéraire: il a alors 60 ans. En 1953, il reçoit le prix de la Culture de Suisse centrale (Kulturpreis der Innerschweiz).

Le manuscrit est prêt en mai 1954. Son éditeur lui demande quelques petits aménagements, notamment revoir des longueurs à certains endroits, ce qu'il accepte, mais il ne cède pas sur le parler local et les régionalismes qui caractérisent ses personnages. Le roman est un succès. Le professeur Karl Schmid, recteur de l'École polytechnique fédérale de Zurich, l'intègre dans son discours de rentrée universitaire ${ }^{(19)}$.

Le roman Urwang constitue une étape importante dans la carrière littéraire d'un écrivain très à l'écoute de la nature, observateur de la faune et de la flore alpestre. Il montre en détail comment une vallée va être transformée pour y installer un barrage hydroélectrique, afin d'augmenter le potentiel économique de la région - avec les conséquences sur la vie humaine (en expropriant les habitants, dont les nombreux paysans pauvres), mais aussi sur la faune et la flore. Le roman, écrit il y a plus de 60 ans, est d'une brûlante actualité.

ich technische Probleme bewältige und kannst Dir vorstellen, was ich mir aufbürde.» Cité d'après B. von MATt, ibid.

15 «Im Frühling will ich ins Göschenertal, das nun auch daran glauben muß, um mit eigenen Augen und Ohren zu sehen und zu hören, wie so etwas vorbereitet wird. Chusch au? Wenn Dir zufällig Material über damit zusammenhängende Probleme der Expropriation und Neuansiedlung in die Hände kommt, so notiere bitte für mich, wo es zu finden ist.» Ibid.

17 Ibid., p. 240.

18 Ibid., p. 238.

19 Ibid., p. 240. Le titre du discours du recteur Karl Schmid est: "Zur Armut gehört die Klugheit». 
Le lieu "Urwängi», qui a inspiré à Inglin le titre du roman, est une vallée située sous la chaîne des Nidwaldner jusqu'aux Urnerbergen, que l'écrivain voyait depuis la chambre où il travaillait ${ }^{(20)}$ à Schwyz:

"Von Meinrads Stube “im Grund” überblickt man die ganze Kette der Nidwaldner - bis zu den Urnerbergen. Als markanter Gipfel ragt der Oberbauen hervor. Als wir einmal von diesen Bergen sprachen, sagte mir Meinrad: "Dort, am Fuß der höchsten Wand des Oberbauens, liegt die Alp Urwängi. Dieser Name hat mich zum Titel des Romans 'Urwang' angeregt. Ich bin zweimal von Bauen her dort hinaufgestiegen, um dieses 'Urwängi' zu sehen. Der Klang des Namens hat mich gelockt."

Er lockte auch mich. Die Alp liegt in einem gewaltigen, steinigen Kessel, hart unter der Bauenwand. Am Rand dieses Kessels, dort wo man fast senkrecht tief unten das Dunkelblau des Urnersees erblickt, stehen Hütten und Gaden » ${ }^{(21)}$.

Le roman suggère et montre un lien intuitif entre les hommes et la nature, les animaux comme les plantes. Il contient aussi, à l'image de l'œuvre d'Inglin, des descriptions de chasses automnales ${ }^{(22)}$, de paysages alpestres fabuleux (dont celles de pentes vert doré et brillantes au soleil), de la couleur des forêts d'automne, du brouillard de septembre ou de la lumière de fin d'été (des tableaux dignes de peintres naturalistes) et de détails qui ne trompent pas un œil avisé, comme celle d'un érable sycomore. Il soigne la construction de ses paysages, comme dans tous ses romans la nature est proche du lecteur, à portée de main. Il veut éviter d'instrumentaliser la nature et donne une copie de la nature aussi naturaliste que possible. En miroir, on trouve la politique économique qui se joue de tout, loin du terrain et du quotidien, de l'humain et des besoins: toujours à la recherche du profit, elle écrase tout. Le roman montre de façon précise comment on brade le paysage pour le profit de quelques-uns et la prospérité d'une région.

Inglin démontre ainsi que l'engagement citoyen est primordial pour lui. Pas d'écriture sociale sans position citoyenne. Beatrice von Matt, sa biographe, caractérise sa position comme une littérature engagée à chaque ligne, profondément en colère, mais lucide et toujours très poétique. Inglin ne fait pas de peinture de genre, en noir et blanc, avec les bons d'un côté et les mauvais de l'autre. Il montre, par des nuances, une situation difficile et délicate, où tous seront finalement perdants. Il parvient à faire couleur locale dans la présentation des personnages: les paysans parlent dans leur dialecte, comme si on y était.

Le roman est constitué de 33 chapitres, le temps de l'histoire dure un peu plus d'une année et demie, le scénario commence par un beau matin de juin ${ }^{(23)}$, les chapitres 15 à 18 se déroulent l'été suivant. La construction du roman est bien symétrique. Le roman débute in medias res avec le personnage principal, Major Bonifaz von Euw, qui apparaît accompagné de sa chienne teckel Sophie. Le décor champêtre accueille le

20 Sa chambre de travail se situait dans l'auberge Wysses Rössli, qui lui mettait à disposition cet atelier de travail en ville (seine Stube). Il y prenait aussi ses repas et y habitait en hiver, car sa maison était difficile à chauffer. Elle se trouve dans l'actuel hôtel Wysses Rössli, sur la place centrale de Schwyz. 
lecteur, le pont en bois qui permet l'accès à la vallée ouvre la perspective de manière théâtrale sur le site controversé.

Peu à peu on découvre les habitants, notamment Franziska qui suspend son linge, la chapelle qui est témoin de tant de soupirs et qui va disparaître inondée (chap. 2), l'idylle entre Franziska Steiner et Lieni Ulrich et leurs projets d'avenir pour une vie commune (2). Les dialogues des personnages suggèrent les très précaires conditions de vie des habitants de la vallée, les conditions économiques difficiles (pas de travail, ou alors très peu), les difficultés sanitaires (accès aux soins et traitement des personnes âgées), la situation des plus chanceux (le fils de l'aubergiste, entre autres), la concurrence entre les hommes et les familles, les tensions, les combats, les jalousies, les mesquineries, les comportements de bassesse (en point d'orgue, le vol de la statue de la vierge dans la chapelle, 30), les classiques fuites dans l'alcool (4). La découverte du projet est filée à travers des discussions sur l'état de la nature (5) et de la chasse locale. Le roman suggère aussi la consanguinité des habitants de la vallée (6). Certaines scènes sont des morceaux d'anthologie, telle celle de Steiner, l'aubergiste, qui œuvre pour la construction du barrage, voyant une promesse de grand profit pour son établissement par l'afflux supputé de touristes en masse. Au moment où il se couche saoul dans son lit, repose à côté de lui son père mort $(8)^{(24)}$. Steiner, dans son avidité pour le profit, ne connaît que l'aspect linéaire du temps, il n'en connaît ni l'aspect cyclique ni l'aspect eschatologique $^{(25)}$, la dimension spirituelle lui échappe complètement, comme le suggère Inglin dans l'ironie de cette situation tragi-grotesque. On assiste aussi à la vente en direct de fermes aux promoteurs (celle de Steiner, dont l'épouse est fille de paysans, 13), au travail de conception des ingénieurs qui préparent le projet de construction du barrage $(14,21,23)$. Le lien entre la vie domestique et la vie spirituelle est mis en avant (15), la chapelle joue un rôle important dans le lien social et se trouve au cœur de festivités, alors qu'elle va disparaître. La situation de la jeunesse locale est préoccupante: entre les amours déçues, les attentes frustrées, les espoirs et la fuite dans l'alcool et le nouveau dieu radiophonique, le malaise des jeunes est palpable. Des scènes poignantes de vérité permettent de présenter des avis et des perspectives différents (la mise au point de Mme Steiner à son mari, qui se veut être un homme de progrès, mais avide de profit, espérant pouvoir changer de situation professionnelle et augmenter son statut social, alors que son couple est en déroute (19)).

Le suspense et la pression augmentent lentement au fur et à mesure du déroulement chronologique des événements. La veille du départ (abandon des maisons expropriées), une scène difficile montre Daniel qui se rend pour la dernière fois à la chapelle (21). Le lecteur avance pas à pas, comme si on y était, découvrant aussi les tractations pour l'abandon des maisons et des terres, les pressions qui sont mises en œuvre pour ceux qui ne sont pas volontaires dans la cession de leurs biens, l'absence de démocratie

24 «Der Vater hat seinen fortschrittlichen Sohn weit hinter sich gelassen. Er war lange ein rückständiger, verbitterter Bauer gewesen, aber jetzt lag er, unantastbar in seine Würde entrückt, neben dem kläglich Lebendigen vollendet da.» Ibid., p. 55. Dans ce chapitre, on découvre aussi le droit d'expropriation sur lequel Inglin s'était bien documenté.

25 Voir, pour la problématique du temps de la vie et de l'au-delà, la dimension spirituelle et la spiritualité, l'étude de Daniel Annen, "Wie ein Buch prophetisch werden kann. Meinrad Inglins Roman Urwang neu aufgelegt», in: Grüße aus der Kantonsschule Kollegium Schwyz, 3/9, déc. 2009, p. 25-28, ici p. 28. 
à Urwang (23), les doutes finaux des plus convaincus, l'abattement de ceux qui ont résisté, la frustration et les interrogations sur un avenir ailleurs.

Le personnage de Steiner devient le Judas du roman, car le progrès est plus important pour lui que l'avenir ou le sauvetage de la vallée. La modernité quelle qu'elle soit lui importe plus que son appartenance à une affiliation spatiale territorialement délimitée et limitée. Son avenir est présenté avec ironie, car lui aussi fait le jeu des prospecteurs et des investisseurs. Alors qu'il pensait pouvoir tirer profit de la situation de l'implantation du barrage, il s'avère que son auberge sera aussi noyée sous les eaux et qu'il est perdant au même titre que ceux qu'il a trompés. Le sort se joue de lui, qui pense linéairement, alors que ses collègues paysans vivent de manière cyclique le chamboulement du temps; ils sauront comment se positionner dans un avenir qui ressemble avec amertume à un passé déjà vu ou vécu. On retrouve dans ce roman une figure de proue dans celle du Major qui ne fait pas office de Sauveur, mais plutôt de père spirituel, de Seigneur féodal qui domine intellectuellement les paysans de la vallée qui l'entourent, qu'il respecte et auxquels il est attaché. Les circonstances font de lui un personnage de regrets qui s'attire la pitié du lecteur, car en définitive il sera lui aussi une victime de la marche des événements, malgré son statut particulier.

Le roman permet aussi à l'auteur de présenter ses doutes sur les techniques de chasse, le bien-fondé de celle-ci, la protection de la nature dont il se fait le chantre. Le roman devient également, à travers la destruction programmée et détaillée, le reflet d'une société humaine aux conditions de vie précaires et dont l'avenir est présenté comme incertain et aucunement meilleur.

Mais le roman montre aussi de belles scènes de nature imprenable, la présence du brouillard et son manteau opaque qui englobe toute la nature pour la transformer en paysage merveilleux, l'avancée des saisons, des scènes de chasse avec des détails comme si on y était (qui aujourd'hui ne font plus rêver les lecteurs!). La beauté sauvage de la nature contraste avec la déroute annoncée de la société humaine. Inglin travaille le paysage de manière réaliste, en observateur, mais aussi en lecteur. Dans ses archives, on trouve des coupures de presse sur différents sujets, tels que l'éloge du brouillard, du vent, la chute des feuilles en automne, les prémices du printemps, des considérations sur la nature, l'homme et la technique, l'eau, la terre, l'air, la lumière, la rivière, la nature et les sentiments, etc. ${ }^{(26)}$.

Le sacrifice du site naturel illustre l'engagement d'Inglin pour la protection des sites historiques et naturels dans des régions qui lui tiennent à cœur dans sa patrie. Dans le contexte d'industrialisation forcenée et de déconstruction des paysages naturels à des fins spéculatives et de profits, le roman résonne comme une parabole ${ }^{(27)}$ qui ancre dans la littérature des faits qui se sont répétés plusieurs fois. Il apparaît comme une tentative d'exorcisation de démons que l'écrivain ne peut plus supporter, mais également

26 Cf. NIZ 4018 - Natur 1.) Natur (allg., Naturbegriff). Ce dossier montre aussi des coupures de presse et des documents sur les catastrophes naturelles, tels le glissement d'un pan de montagne à Goldau, des avalanches, des tremblements de terre, des glissements de terrains et de roches en rapport avec la vie des hommes, etc. 
comme un engagement sur le plan littéraire d'un écrivain dont l'engagement sociétal s'était cantonné jusque-là à sa vie privée. Cet engagement littéraire lui a coûté, il a du mal à surmonter ses scrupules, qu'on lit dans sa correspondance, mais cela ne l'empêche pas d'engager davantage sa personne pour la préservation de la nature ${ }^{(28)}$. L'engagement sociétal d'Inglin pour une cause qu'il tient pour juste est peu commun dans sa génération, mais certains écrivains, et non des moindres, prêtent leur plume et leur voix à des causes similaires. Beatrice von Matt souligne les prises de position de Günter Eich («Rede vor den Kriegsblinden», $1953^{(29)}$ ) où ce dernier évoque la mécanisation croissante de la vie et l'automatisation qui l'accompagne, qui déferle sur la société tel un totalitarisme, et la disparition des valeurs. On entend les mêmes accents chez Inglin, à l'occasion de la fête de son soixantième anniversaire et aussi dans le discours de réception du Kulturpreis der Innerschweiz ${ }^{(30)}$.

Les critiques, et en premier lieu Emil Staiger, seront sensibles à la sobriété de la langue d'Inglin et à sa précision factuelle. Staiger établit très justement un parallèle avec le récit Heimat de Jakob Bosshart (1914, paru dans le recueil Erdschollen), un écrivain avec lequel Inglin a de nombreux points communs, tels les descriptions naturalistes, la lucidité sans pathos, la constatation d'un monde en train de disparaître, le changement ou la perte des valeurs ancestrales, les changements en cours dans la société, la perte de repères, le recul de la spiritualité - autant de sujets qui sont aujourd'hui d'une actualité certaine.

La préparation du roman demande une grande documentation, il étudie ainsi des relevés chiffrés et des cartes des vallées suisses inondées, étudie également des procédures d'expropriation menées par les ingénieurs ${ }^{(31)}$. Ces documents lui permettent de proposer dans le roman des faits très réalistes et très précis, qui lui confèrent une facture de manifeste très crédible. Il ancre son travail dans une réalité tangible, la littérature devenant en quelque sorte une copie de la vie réelle, dans un exercice de recomposition. On retrouve dans ce besoin de grande précision l'attitude d'Inglin lors de ses sorties en randonnées, où il ne laissait rien au hasard et consultait régulièrement ses cartes, pour rester efficace et optimal (par exemple pour la recherche de zones de repos). On trouve dans ses archives des documents manuscrits qui recensent des surfaces de prairies, des têtes de bétail, de moutons, également les mensurations de vallées (longueur, largeur et profondeur), le croquis manuscrit d'une vallée et celui des lieux du roman avec la rivière Aa en couleur bleue. La structure du roman y est également présentée sous forme de relevé détaillé par chapitre. Malgré la factualité du texte, la langue reste d'une grande évocation poétique, et les sentiments humains qu'il met en

28 Ibid., p. 239 et 293. Inglin a obtenu une autorisation policière de surveillance de sa région pour la préserver du pillage floral des randonneurs. Habilité à contrôler les sacs à dos de randonneurs, il a ainsi contrôlé un groupe de jeunes gens dont le sac contenait des edelweiss et, à une autre occasion, des gentianes.

29 Ibid., p. 239.

30 Ibid.

31 L'inspiration de ces cartes se lit dans certains bulletins de sociétés dont il est membre, par exemple Schweizer Naturschutz/Protection de la nature, Bâle, $\mathrm{n}^{\circ}$ 1, février 1951, en p. 11, la carte des barrages en projet dans les Grisons. L'article montre que des intérêts financiers privés se sont constitués en lobbies. 
scène sont touchants de vérité et de fragilité, tout comme les mouvements de la nature, des éléments en montagne et l'évocation de la vie animale.

Le travail mené sur la composition du roman est important et très engagé. La présence très marquée du dialecte régional ancre le roman encore davantage dans une réalité quotidienne tangible. Inglin se défend et se justifie; on laisse la connotation dialectale en l'état et en dépit de ces craintes le succès sera au rendez-vous.

La presse suisse salue unanimement la parution du roman comme un document sérieux, tangible, bien étayé et novateur, visionnaire aussi. Le professeur Karl Schmid l'intègre dans son discours inaugural de président d'Université pour la rentrée universitaire (13 novembre 1954) ${ }^{(32)}$. Son ami Traugott Vogel salue cette parution ainsi, en insistant sur le ton et la mesure par rapport à la catastrophe que dévoile le roman:

«Ich nahm mir wieder Dein schönes, ernstes Buch vom Stausee vor, las wieder darin und begab mich nochmals in Deinen Bann. Ich las es zum zweiten Mal eben fertig und lasse die schöne Erregung nicht abklingen, bevor ich aus ihr heraus Dir ein paar Dankes- und Bewunderungsworte gegeben habe. Neben vielen, treuen, das geistige Aroma bestimmenden Herrliche Einzelheiten, von denen ich am liebsten einige aufzählen möchte, um von Dir zu hören, ob sie Dir auch so gefallen wie mir, - [...] also neben den tonangebenden Einzelheiten (die im Antlitz des Werkes die Physiognomie bestimmen), liebe ich die Melancholie, diese mannhafte Trauer, aus der diese Insel auftaucht und in sie versinkt. Überhaupt Dein Maß! Dein Maßhalten in der Klage! » ${ }^{(33)}$.

Dans une lettre du 19.12.1954, son ami Hans von Matt montre beaucoup de compréhension pour la situation de dessaisie décrite par Inglin dans Urwang, car lui-même vit une situation personnelle analogue avec la construction d'une école et d'un magasin juste devant son garage, ce qui défigure le paysage naturel et l'oblige à entreprendre des travaux pour pouvoir à nouveau avoir accès à la rue: «Ich komme mir wirklich vor wie einer der Bauern aus deinem Urwangtal. Man streubt sich, man wehrt sich, aber unabwendbar kommt das Verhängnis. » Plus haut, il cite Kafka, à juste titre: «Die Art, wie das Verhängnis über diese unschuldigen Menschen hereinbricht, gemahnt an Kafka. Dieses nutzlose sich Wehren bis zum letzten, diese Zuflucht zu immer kleineren Mitteln, die immer weniger nützen.»

Lors d'une randonnée avec le même Hans von Matt en 1967, les deux amis déplacent des orchidées qui sont en perdition sur une pente de montagne en train de s'effriter. Hans von Matt fait le parallèle avec la fin du roman Urwang:

«Im Sommer 1967 durchstieg ich mit Meinrad auf einem schmalen Weglein das Tobel, um ihm auf einer steil abfallenden Kante jene Stelle zu zeigen, wohin wir vor einigen Jahren mehrere Stöcke des roten Waldvögeleins versetzt hatten. Ein nicht viel denkender Bauer hatte weiter unten einen breiten Weg in jenen Abhang geschnitten, der diese roten Waldvögelein und andere Orchideen trug. Die ganze Halde oberhalb des Anrisses drohte nun abzurutschen. Bereits waren Teile davon abgerissen und lagen auf der beabsichtigten Straße. Wir sahen voraus, daß die Bauern im Herbst an Stellen ihrer Straße einen

32 Il y parle notamment de «messianisme technique», in: B. von MatT, Meinrad Inglin (note 13), p. 240. La même année paraît le fameux roman Stiller de Max Frisch, autre écrivain de la jeune génération qui affirme sa différence et que Schmid intègre pour cette raison dans son discours.

33 Meinrad Inglin, Die Briefwechsel mit Traugott Vogel und Emil Staiger, herausgegeben und mit Anmerkungen versehen von Felix R. Hangartner, Zurich, Ammann, 1992, p. 147. 
Muhrgang haben würden, und wir auch. So retteten wir einige dieser hübschen, großblumigen Orchideen und verpflanzten sie an diese sichere, vor Blicken, verborgene Stelle, wo schon andere Waldvögelein angezeigt hatten, daß es ihnen dort behage.

Meinrad blieb stehen und betrachtete die Blumen. Ich wußte, was er dachte. Das war die leibhaftige Parallele zum Schluß seines Urwang-Romans » ${ }^{(34)}$.

Inglin voit son roman comme un exemple ${ }^{(35)}$ lié au développement économique débridé de l'après-Seconde Guerre mondiale. En Suisse, comme dans toute l'Europe, l'augmentation de la production électrique passe par le développement de l'hydroélectricité et des pans entiers de vallées sont sacrifiés à ce besoin économique ${ }^{(36)}$.

\section{Un engagement citoyen en toute logique}

Inglin est très affecté par les projets hydroélectriques, la disparition des vallées sous les eaux, celle de sites historiques et naturels remarquables. Il se lance en 1952 dans la collecte de signatures à Schwyz avec son ami Dr. Hermann Stieger. Il envoie les formulaires remplis à son collègue Stiefel à Winterthur, il montre par là aussi que la nature-mère n'est pour lui pas uniquement une préoccupation littéraire. Il considère l'utilisation technique des forces de la nature comme une profanation (Schändung). Son engagement citoyen a commencé dans sa jeunesse. En effet, il a publié entre 1914 et 1920 dans des journaux des articles contre le projet de barrage du Wägital Werk ${ }^{(37)}$.

Dans les années 1950-52, Inglin proteste contre un projet de téléphérique (Seilbahn) à Schwyz qui devait mener jusqu'au site du Gründel, qui sera abandonné, mais aussi contre le projet d'élargissement de la route qui traverse le col de Pragel (Pragelstrasse ou Muotastrasse). Cette route relie la vallée de la Muota du canton de Schwyz à celle de Klön dans le canton de Glaris ${ }^{(38)}$. Le col se situe sur les communes de Muotathal et Riedern, à une hauteur de $1548 \mathrm{~m}$. Avec des côtes à $18 \%$ et des passages à voie unique, cette route est fermée une partie de l'hiver. La partie de route côté Glaris a été construite en 1941, celle côté Schwyz en 1974. Elle a servi durant la guerre pour des transports de munitions. La route est de par sa pente et sa configuration assez dangereuse et depuis 1978 une partie de sa rampe est fermée à la circulation des motos en fin de semaine. Inglin s'est engagé dans ce combat qui était perdu d'avance ${ }^{(39)}$.

D’autres mobilisations se préparent contre des projets de centrales hydroélectriques. Comme il a l'habitude de le faire, Inglin se documente de manière précise sur tous

$$
\text { ou }
$$
ou Peter Handke, entre autres, s'en prendront à tous ceux qui défigurent les paysages alpestres pour
donner accès à une masse de touristes aux sommets (les pistes de ski, les paysages préservés dans les Alpes autrichiennes).

37 Cf. B. von MAтT, Meinrad Inglin (note 13), p. 237. Ce barrage se situe dans le canton de Schwyz et fut construit en 1925.

38 Voir notamment www.e-periodica.ch/cntmng?pid=geo-007:2007:105_1238-d.pdf et aussi: www. admin.ch/opc/de/federal-gazette/1939/index_11.html [25.06.19].

39

Cf. B. von MAтT, Meinrad Inglin (note 13), p. 237. 
les aspects du problème, dont entre autres la production d'électricité ${ }^{(40)}$. Le sujet est brûlant à cette époque et les journaux préparent l'opinion publique, par exemple par des dossiers thématiques comme le numéro spécial du NZZ du 11.06.1953 intitulé «Elektrizität in Wirtschaft und Technik», dans lequel sur une demi-page on trouve la photo d'un grand torrent ${ }^{(41)}$. Il est question de la résolution du problème de l'approvisionnement en énergie de la Suisse qui dépendait à $62 \%$ de l'import. Les études balaient tous les domaines de cette production d'énergie, par exemple «Ausverkauf der Schweizer Wasserkräfte» du Dr. h.c. Henri Niess, ingénieur SIA (Baden) dans le $N Z Z$ du 11.06.1953. Le côté financier n'est pas ignoré: "Betrachtungen über Einheitstarife» dans le NZZ du 11.06.1953 du Dr. W.L. Froelich (Zurich); on alterne aussi les perspectives, par exemple "Thermische Kraftwerke im Dienste unserer Elektrizitätsversorgung», $N Z Z$ du 11.06.1953 ou, pour changer de ton et montrer une autre perspective, dispendieuse, «Energiehungrige Weltwirtschaft» du Dr. E. Waldmeyer (Berne) également dans le NZZ du 11.06.1953.

Mais son intérêt date de plus de dix ans, où il a suivi par la presse l'évolution de la situation sur l'exploitation des forces naturelles en Suisse «Ausnutzung der Schweizer Wasserkräfte», NZZ, 04.09.1945, «Der Ausbau unserer Wasserkräfte», NZZ, 06.10.1945, «Das Wasserschloss Graubünden », NZZ, 23.06.1945. Auparavant déjà on lit son intérêt pour "Das Rheinwald-Kraftwerk» dans le fascicule Schweizer Naturschutz/Protection de la nature, Bâle, $\mathrm{n}^{\circ} 4$, juin 1942. Il s'agit du projet de construction d'un barrage hydroélectrique à Rheinwald dans les Grisons ${ }^{(42)}$. Malgré les promesses de gain d'argent et les intimidations à l'égard des trois villages concernés Splügen, Medels et Nufenen (avec des arguments de type: «toute la Suisse aura plus de lumière, de courant, de chaleur et de travail si les habitants déménagent»), les villageois ont tenu bon ${ }^{(43)}$. En 1945, ils ont refusé à $95 \%$ le projet, si bien qu'un an plus tard le gouvernement fédéral à Berne s'est vu contraint de mettre fin au projet. Cette victoire est rare dans les annales suisses et elle mérite de ce fait d'être soulignée!

40 NIZ 4003 2.) 4009, voir aussi l'article du NZZ du 29.08.1953, intitulé «Vom Stand unserer Elektrizitätsversorgung», ou encore celui du même NZZ du 21.07.1954, intitulé «Der Export elektrischer Energie» de Ch. Aeschlimann (Olten). Sa préoccupation pour le sujet de la production d'énergie est ancienne, voir aussi un autre article du NZZ du 03.11.1930 (sur les forages pétroliers d'Oklahoma, qu'on retrouve aussi dans «Erdölfunde aller Welt» dans le $N Z Z$ du 26.03.1960). Peu à peu, c'est la préoccupation pour l'énergie atomique qui s'installe, $N Z Z$ du 22.01.1954 sur «Der große Atombericht», où l'énergie nucléaire est présentée comme celle de demain, également dans «Atomkernspaltung und Energieversorgung der Zukunft», NZZ du 24.03.1954. Ou encore «Energieerzeugung und Atomkraftwerkbedarf in der Schweiz», NZZ du 11.10.1954, et plus globalement «Zukunftsfragen der Schweizerischen Energiewirtschaft», NZZ du 06.09.1953.

41 L'auteur en est le Pr. Dr. Bruno Bauer de l'École polytechnique fédérale de Zurich. Les spécialistes sont sollicités pour accréditer ces études.

42 www.heimatschutz.ch/uploads/tx_userzeitschrift/1_2018_3df_02.pdf et aussi www.bernerzeitung. ch/schweiz/standard/sufers-und-der-truegerische-glanz-des-fluessigen-goldes/story/11345005 (consulté le 25.06.2019). Le projet du Splügner Stausee devait mettre sous les eaux 5,5 km² de paysage, dont 300 ha de prairie, 100 ha de forêt et 3 villages.

43 Un autre projet, celui dans la vallée d'Urseren (1947-50), aura lui aussi capoté par l'initiative citoyenne et la résistance des habitants locaux. Ces deux échecs sont évoqués brièvement par le Major dans le roman. Voir, pour les explications détaillées sur ces projets, M. INGLIN, Urwang (note 22), « Nachwort von Ulrich Frei», p. 273-288, ici p. 273-274. 
Durant l'hiver 1952, Inglin collecte des signatures à Schwyz contre le projet de barrage hydroélectrique sur le Rhin (Rheinau Initiative). À partir de 1952, un projet de barrage de retenue sur le Rhin à Rheinau près de Schaffhausen mobilise à nouveau les consciences écologiques. Inglin rédige des articles, dont certains sont anonymes, contre le Rheinaubund. Il s'engage dans une collecte de signatures contre ce projet, obtient de l'aide du Dr. Hermann Stieger et envoie les formulaires de pétition remplis à son ami Stiefel, un combattant de la première heure, à Winterthur ${ }^{(44)}$. Ce combat affecte Inglin en profondeur, car il ne supporte plus qu'on touche à l'intégrité de la nature. De ce fait, il vit cet épisode comme une souillure. Il se mobilise avec un cercle d'amis, dont Traugott Vogel, à qui il écrit le 2 mai 1952:

«Du hast für die Erhaltung der Rheinau Deinen Namen gegeben. Bravo! Gern hätte ich den Aufruf auch unterzeichnet, aber ich bin nicht angefragt worden. Die Petition gegen das Werk habe ich mit Überzeugung unterschrieben und hier auch Unterschriften dafür gesammelt» ${ }^{(45)}$.

Dans leur correspondance, le sujet revient de temps en temps, Vogel s'engage en public et dans la presse (lettre du 17 novembre 1953):

«[...] morgen werde ich mit ein paar Worten meiner Empörung, stärker noch meiner Trauer über die Rheinau-Schändung Ausdruck geben können. Ich warte auf den Augenblick der Entladung wie auf eine Erlösung; ich bin sehr bedrückt, da mit diesem Stück Heimat ein Teil meiner Selbst defloriert wird. Und über solchen Verlust hilft nur öffentlicher Schmerzausbruch lindernd hinweg (Laokoon!)» ${ }^{(46)}$.

Inglin se constitue, comme à son habitude, un dossier technique sur le sujet. Dans le $N Z Z$ du 22.01.54, on lit le fonctionnement politique du projet et les instances concernées. Il y est même donné des précisions sur les agences pour l'emploi (Arbeitsämter) de Zurich, Schaffhausen et Waldshut, qui embauchent plus de travailleurs immigrés, car ils ne disposent pas d'assez de main-d'œuvre locale. Il consulte aussi le livret «Kraftwerk Rheinau» (Entscheid des Schweizer Bundesrates 24.06.52). Dans sa correspondance, on suit les étapes du déroulement des événements. Inglin participe aux publications contre la construction de ce barrage, avec ses amis il participe à la rédaction des textes des tracts qui sont distribués, à la discussion de l'organisation des opérations (type de tracts, nombre, distribution, etc.), et surtout à la résolution de la question financière (en participant à la prise en charge de cette opération, lettre de Hermann Stieger du 28.12.58). Le projet suit son cours et l'année suivante, Vogel récidive (lettre du 9 mars 1954):

«Sie sollen die Rheinau schänden: Der Fluch wird über sie kommen, und wir werden klagen: Und ich begehre, nicht schuld daran zu sein!

Ich glaube mit Dir, lieber Meinrad, daß uns heute kein edleres, dringlicheres, befeuernderes Motiv gegeben ist als dieses : vor den Naturschindern zu warnen, sitzen sie wo sie nur wollen, und ihnen zu sagen: Es kann euch nie verziehen werden, denn ihr wißt, was ihr tut!

An Deiner Seite bleibe ich zuversichtlich. Ich bin Dir von Herzen dankbar [...] ${ }^{(47)}$. 
Inglin reste sur ses gardes et surveille l'évolution de la situation qui tourne hélas en défaveur des militants. Une consultation publique sous forme de votation est organisée dans la région concernée; il écrit à ce propos à son ami Vogel (lettre du 13.10.1954) son dépit face à l'évolution de la situation et son incapacité viscérale, en tant qu'intellectuel, à faire face socialement à ce problème:

«Mich bedrängt die bevorstehende Abstimmung über die Rheinau-Initiative. Das überparteiliche Komitee schickt mir Material zur Propaganda, das ich hier umsonst an den Mann zu bringen versuche. Wo ich anklopfe, zuckt man die Achsel. Der Schwyzer Ständerat Dr. Auf der Mauer, mit dem ich befreundet bin, vertritt natürlich auch hier den Standpunkt, den er als Präsident der ständerätlichen Kommission eingenommen hat, und verlangt entschlossene Ablehnung. Ihm wird man folgen, während man mir meinen vermutlich ungeschickt vertretenen gegenteiligen Standpunkt nur eben freundlich verzeihen wird. Es wurmt mich, daß ich dieser praktischen Bewährungsprobe nicht recht gewachsen bin, und nur mein neues Buch [i.e. le roman Urwang] tröstet mich noch, (das Du im November erhalten wirst,) und mit dem ich den Auftrag zu erfüllen hoffe, der uns in dieser Beziehung gestellt ist ${ }^{(48)}$.

La réaction de son ami face à sa détresse et ses doutes ne tarde pas à arriver (lettre du 16.10.1954): Inglin a apporté par son roman, à ce combat et à tous les autres de la même sorte, une contribution magistrale qui fait date, et son engagement se lit dans son œuvre littéraire de premier plan:

"[...] Was die Sache Rheinau angeht, hast Du ganz gewiß mit Deinem neuen Buche mehr als jeder Redner getan. Du darfst nicht von einer Bewährungsprobe reden, der Du nicht recht gewachsen seiest; man hat Dich als Erzähler auf solche Werbetätigkeit überhaupt nicht zu verpflichten. Dein Name und Dein Buch genügen; Soll etwa der Fähnrich mit deiner Fahnenstange noch drauflosstechen? (Und daß die Rheinau noch zu retten sei, darf man ja kaum noch hoffen - bei dieser Stumpfheit unserer rasenden maroden, von der Technik besessenen Zeit!) » ${ }^{(49)}$.

La mobilisation fut très importante, elle vint de toute la Suisse. Curieusement, c'est la votation locale qui porte le coup de grâce à cette belle résistance: les lobbies et le profit ont eu raison de la beauté du site ${ }^{(50)}$.

Fin 1955, Inglin participe au combat contre la construction de la centrale hydroélectrique de Zurich, située près de Schindellegi-Hütten ${ }^{(51)}$. Dans cette action, son ami le professeur Emil Egli tente de le convaincre de prendre position en public, mais Inglin

48 Ibid., p. 139-140.

49 Ibid., p. 141.

50 Près du barrage construit sur le Rhin se trouve l'île de l'abbaye de Rheinau, le paysage y était, avant la construction du barrage, très romantique. Cf. Gedenkschrift zur 1200 Jahrfeier (AK 4241), voir notamment le chapitre sur «Das Kraftwerk Rheinau», p. 95-104.

51 Cf. NI M 17 Naturschutz 3.)3.5.)-3.7.) et aussi NI M 17 Naturschutz 3.)3.8.)-3.9.), où une lettre du 14.01.1956 montre l'engagement de la Natur- und Heimatschutzkommission des Kantons Schwyz, dont Inglin est trésorier, contre le projet de cette retenue sur la Sihl, un affluent de la Limmat. Le compromis proposé par cette association semble n’avoir pas été lu par les auteurs du projet. Celui-ci fut mené par l'entreprise qui produit de l'électricité, dont le prix devait être divisé par deux. Inglin constate encore une fois que le profit prime sur l'avis des habitants. Le fonds d'archives montre l'importante collecte de documents et de témoignages qu'Inglin a amassés, par exemple «Gefährdete Sihllandschaft» von Dr.h.c. Walter Höhn-Ochsner, NZZ, 16.12.1955. 
s'y refusera toujours. Il sait qu'il n'est pas un bon orateur, qu'il entend mal et qu'il n'a pas la capacité de répondre instantanément dans des joutes publiques. Il est prêt à engager son nom et sa réputation, mais il se sait être un mauvais acteur (lettre à Emil Egli, 28.10.1953 ${ }^{(52)}$ ). Tout comme Inglin, on a l'impression, en lisant sa documentation, que la presse est très orientée vers les soi-disant progrès techniques et qu'elle les soutient. De temps en temps, un article prend le contrepied, comme celui du NZZ du 18.04.1963, «Wenn Fische und Bäche stimmen könnten...», mais ils sont bien rares.

Pour Meinrad Inglin, il fut difficile de lutter contre l'air du temps, et déception et amertume furent de mise à la fin de sa vie. Urwang est le roman d'une époque de crise et de profonds changements. Il est le testament écologique d'un écrivain rebelle et engagé pour la préservation de la nature alpestre et la vie des hommes, libres du progrès technique. Toute son œuvre montre le côté maternel et originel de la nature. Chez Inglin, la destruction et l'apocalypse viennent de la main de l'homme. Le roman, qui est le manifeste bien documenté d'une catastrophe écologique annoncée, ne fait pas de catastrophisme inutile, mais reste ancré dans un réalisme pragmatique et un engagement sérieux pour la préservation des sites naturels. L'espoir d'une nature triomphante perdure dans son œuvre à travers l'action d'un homme engagé, visionnaire et sincère dans sa relation à la nature et sa croyance en l'avenir de la vie humaine. Sa position est novatrice dans le contexte de son époque et sa génération d'écrivains et elle demeure d'une actualité certaine.

\section{Résumé}

Meinrad Inglin (Schwyz, 1893-1971) est un auteur du terroir suisse, habité par une croyance animiste et panthéiste, très soucieux de la préservation des sites, de la faune et de la flore de son pays natal. Il oppose la vie dans la nature à la civilisation technicienne. Citoyen militant engagé dans des actions de manifestations contre la construction de barrages et de routes en Suisse dans les années 1950-60, il s'est engagé pour la préservation de l'environnement. Le roman Urwang (1954) montre les aspects catastrophiques de l'inondation d'une vallée pour la construction d'un barrage et d'une centrale hydroélectrique. Il constitue un tournant dans son cuvre, Inglin y démontrant que l'individu est quantité négligeable quand des intérêts financiers sont en jeu. Il dénonce dans son roman les catastrophes écologiques induites par la massification du tourisme et l'industrialisation.

\section{Zusammenfassung}

Meinrad Inglin (Schwyz, 1893-1971) ist ein schweizerischer Heimat-Schriftsteller, der von einem animistischen und pantheistischen Glauben beherrscht ist. Sein Interesse gilt der Erhaltung der Natur, der Fauna und Flora seiner Heimat. Er stellt das Leben in der Natur der technischen Zivilisation entgegen. Als Bürgerinitiator, der in den 1950er und 1960er Jahren an Demonstrationen gegen den Bau von Staudämmen und Straßen in der Schweiz teilgenommen hat, setzte er sich für Schutz und Erhalt der Umwelt ein. 
Der Roman Urwang (1954) zeigt die katastrophalen Folgen der Überschwemmung eines Tals zum Zweck eines Staudamms und eines Wasserkraftwerks. Dies entspricht einem Wendepunkt in seinem Werk. Inglin zeigt, dass das Individuum nichts wert ist, wenn es um finanzielle Interessen geht, und er prangert die ökologischen Katastrophen an, die die Massifizierung des Tourismus und die Industrialisierung mit sich bringen.

\begin{abstract}
Meinrad Inglin (Schwyz, 1893-1971) is a Swiss country author, inhabited by an animist and pantheist belief, very concerned about the preservation of the sites, fauna and flora of his native country. He opposes life in nature to technician civilization. A citizen activist involved in demonstrations against the construction of dams and roads in Switzerland in the 1950s and 1960s, he committed to preserve the environment. The novel Urwang (1954) shows the catastrophic aspects of the flooding of a valley for the construction of a dam and hydroelectric power plant. This means a turning point in his work: Inglin shows that the individual is negligible when financial interests are at stake and he denounces the ecological disasters induced by the massification of tourism and the industrial development.
\end{abstract}

